\title{
PLURISUBHARMONIC FUNCTIONS AND CONVEXITY PROPERTIES FOR GENERAL FUNCTION ALGEBRAS
}

\author{
BY
}

\author{
C. E. RICKART ${ }^{(1)}$
}

\begin{abstract}
A "natural system" consists of a Hausdorff space $\Sigma$ plus an algebra $\mathscr{A}$ of complex-valued continuous functions on $\Sigma$ (which contains the constants and determines the topology in $\Sigma$ ) such that every continuous homomorphism of $\mathscr{A}$ onto $C$ is given by an evaluation at a point of $\Sigma$ (compact-open topology in $\mathscr{A}$ ). The prototype of a natural system is $\left[C^{n}, \mathfrak{B}\right]$, where $\mathfrak{B}$ is the algebra of polynomials on $C^{n}$. In earlier papers (Pacific J. Math. 18 and Canad. J. Math. 20), the author studied $\boldsymbol{\Re}$-holomorphic functions, which are generalizations of ordinary holomorphic functions in $C^{n}$, and associated concepts of $\mathscr{A}$-analytic variety and $\mathfrak{A}$-holomorphic convexity in $\Sigma$. In the present paper, a class of extended real-valued functions, called $\mathfrak{A}$-subharmonic functions, is introduced which generalizes the ordinary plurisubharmonic functions in $C^{n}$. These functions enjoy many of the properties associated with plurisubharmonic functions. Furthermore, in terms of the $\mathscr{A}$-subharmonic functions, a number of convexity properties of $\mathbf{C}^{n}$ associated with plurisubharmonic functions can be generalized. For example, if $G$ is an open $\mathscr{A}$-holomorphically convex subset of $\Sigma$ and $K$ is a compact subset of $G$, then the convex hull of $K$ with respect to the continuous $\mathscr{A}$-subharmonic functions on $G$ is equal to its hull with respect to the $\mathscr{A}$-holomorphic functions on $G$.
\end{abstract}

Introduction. In previous papers ([10], [11], [12]) we have investigated certain properties of general function algebras which may be regarded as generalizations or analogues of familiar results in the theory of analytic functions of several complex variables. We continue this study with the present paper in which a number of results for plurisubharmonic functions and associated convexity notions are extended to the general situation.

The setting for our investigations is a system [ $\Sigma, \mathfrak{U}]$ consisting of a Hausdorff space $\Sigma$ and an algebra $\mathfrak{A}$ of complex-valued continuous functions on $\Sigma$. It is always assumed that $\mathfrak{U}$ contains the constant functions and determines the topology in $\Sigma$, i.e. the given topology in $\Sigma$ is equivalent to the "coarsest" or "weakest" with respect to which all functions in $\mathfrak{A}$ are continuous. We also call this the $\mathfrak{U}$-topology. The topology in the algebra $\mathfrak{A}$ is the compact-open topology,

Received by the editors March 31, 1971.

AMS 1970 subject classifications. Primary 46E25, 46J10, 32F05, 32F 15.

Key words and phrases. Plurisubharmonic functions, p-convexity, several complex variables, function algebras, natural systems.

(1) Research supported in part by NSF Grant GP-21193. 
i.e. uniform convergence on compact subsets of $\Sigma$. We shall also assume throughout that $[\Sigma, \mathfrak{U}]$ is natural in the sense that every continuous homomorphism of $\mathfrak{U}$ onto the complex numbers $\mathrm{C}$ is given by evaluation at a point of $\Sigma$. The prototype of a natural system is $\left[\mathrm{C}^{n}, \mathfrak{\beta}\right]$, where $\mathbf{C}^{n}$ is ordinary $n$-dimensional complex space and $\mathbb{B}$ is the algebra of polynomials in $n$ complex variables. Thus, in the case of an arbitrary natural system $[\Sigma, \mathfrak{Y}]$, it is convenient to think of the algebra $\mathcal{U}$ as playing a role analogous to that of the polynomials in the case of $\left[\mathbf{C}^{n}, \mathfrak{\beta}\right]$. Other examples of natural systems are (1) any infinite cartesian product of complex planes, again with the algebra of polynomials, (2) a Ste in manifold with its algebra of global holomorphic functions, and (3) the Gelfand representation of a commutative Banach algebra on its space of maximal ideals.

The first problem here is to obtain, for a general natural system [ $\Sigma$, $\mathfrak{X}$, an appropriate extension of the usual notion of plurisubharmonic function in $\mathbf{C}^{n}$. The analogous problem for holomorphic functions, which leads to the notion of $\mathscr{X}$-bolomorpbic function in $\Sigma$, is dealt with in the papers cited above ([10], [11]). One definition for plurisubharmonic functions in $\mathrm{C}^{n}$ goes as follows: Let $G$ be an open set in $\mathbf{C}^{n}$ and $f$ a function defined on $G$ with values in $[-\infty, \infty)$. Then $f$ is plurisubbarmonic on $G$ provided it is upper semicontinuous and, for every holomorphic map $\eta$ of the open unit disc $D$ of $C^{1}$ into $G$, the composition function $f^{\circ} \eta$ is subharmonic in $D[4$, p. 271]. In the case of $[\Sigma, \mathscr{U}]$, one can define a mapping of the disc $D$ into $\Sigma$ to be "holomorphic" if $a \circ \eta$ is holomorphic in $D$ for each $a \in \mathfrak{U}$. Having done this, one can then formulate a definition of "plurisubharmonic " function on an open set in $\Sigma$ exactly as in the case of $\mathrm{C}^{n}$. Unfortunately, this approach breaks down in general since there may not exist any nonconstant holomorphic maps of $D$ into $\Sigma$. Such is the case, for example, with Stolzenberg's hull without analytic structure [14]. This hull is a compact polynomially convex set in $\mathrm{C}^{2}$ which, along with the algebra of all polynomials on it, constitutes a natural system. In such an example, every upper semicontinuous function would, by default, be "plurisubharmonic". Because this kind of pathology can occur in the general situation, a sharper definition is needed.

Our approach is to obtain the desired class of functions by extending in an appropriate way the class $\log |\mathfrak{U}|$ of functions of the form $\log |a|$, where $a \in \mathfrak{U}$. This process, which is described in the next section, gives the ordinary plurisub-

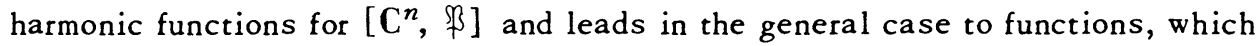
we call "थी-subharmonic", exhibiting many properties analogous to those of ordinary plurisubharmonic functions and providing interesting convexity properties for $[\Sigma, \mathfrak{U}]$. A similar approach is used by Lelong [7] in some of his work on plurisubharmonic functions in $\mathrm{C}^{n}$ and by Bochner and Martin [1, p. 143] in their study of Hartogs' functions. A crucial difference, however, is that our extension process is local in character. The method of obtaining the $\mathfrak{U}$-subharmonic functions by 
extending the class $\log |\mathfrak{Q X}|$ is similar to that of obtaining the $\mathfrak{U}$-holomorphic functions by extending the class $\mathscr{U}$. For this and other reasons, it is desirable to recall briefly the definition of the $\mathfrak{U}$-holomorphic functions.

First let $\mathcal{F}$ be an arbitrary family of complex-valued functions defined on subsets of $\Sigma$. Then a function $g$, with domain of definition $\mathscr{D}_{g}$, is said to be locally approximable by elements of $\mathcal{F}$ if there exists for each point of $\mathscr{T}_{\boldsymbol{g}}$ a neighborhood $U$ such that $g$ is uniformly approximable on $U \cap \mathscr{I}_{\boldsymbol{g}}$ by elements of $\mathfrak{F}$. The collection of all functions that are locally approximable by elements of $\mathcal{F}$ is called the local extension of $\mathcal{F}$ and denoted by loc $\mathcal{F}$. It is obvious that $\mathcal{F} \subseteq \operatorname{loc} \mathcal{F}$. If $\mathcal{F}=$ loc $\mathcal{F}$, then $\mathcal{F}$ is said to be locally closed. Although the local extension of $\mathcal{F}$ need not be locally closed, it is easy to see that there always exists a smallest locally closed family that contains $\mathcal{F}$. We call this the local closure of $\mathcal{F}$ and de-

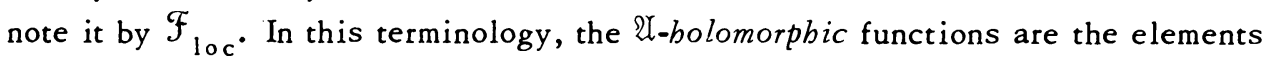

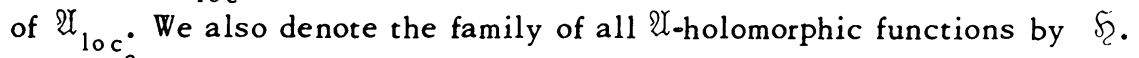

In $\$ 1$ we introduce a general closure operation on subsets of the upper semicontinuous functions in $\Sigma$ which, when applied to $\log |\mathfrak{U}|$, gives the $\mathfrak{U}$-subharmonic functions. Many of the basic properties of these functions are established along with the fact that they reduce in the special case of $\mathrm{C}^{n}$ to the ordinary plurisubharmonic functions. In $\$ 2$ various maximum properties of $\mathscr{U}$-subharmonic functions are established, the most important of which is a local maximum principle. In $\$ 3$ the notion of $\mathfrak{A}$-subharmonic convexity for a subset of $\Sigma$ is introduced and some of the properties of such sets are obtained. In $\$ 4$ the connection between $\mathfrak{U}$-subharmonic convexity and $\mathfrak{U}$-holomorphic convexity is investigated.

1. U-subharmonic functions. The functions that we shall consider are defined on arbitrary subsets of $\Sigma$, take values in the extended real numbers $[-\infty, \infty)$ (i.e. the value $-\infty$ is admitted while $+\infty$ is excluded), and are upper semicontinuous (u.s.c.). We denote by $\mathcal{U}$ the class of all such functions. Note that $\mathcal{U}$ is obviously closed under multiplication by nonnegative reals and under restrictions, i.e. if $f \in \mathcal{U}$ and $X \subset \mathscr{D}_{f}$, then the function $f \mid X$ obtained by restricting $f$ to the set $X$ belongs to $\mathcal{U}$. Also $\mathcal{U}$ is "closed under addition" in the sense that, whenever $f, g \in \mathcal{U}$ and $\mathscr{D}_{f} \cap \mathscr{D}_{g} \neq \varnothing$, the function $f+g$ defined on $\mathscr{D}_{f} \cap \mathscr{D}_{g}$ belongs to $\mathcal{U}$. Thus, with the obvious convention, we can say that $\mathcal{U}$ is "closed under linear combinations with nonnegative real coefficients'.

We introduce next the class $\mathfrak{Q}_{\mathfrak{q}}$ of all functions $f$ of the form $f=n^{-1} \log |a|$, where $n$ is a positive integer and $a \in \mathfrak{U}$. Obviously, $\mathscr{L}_{\mathfrak{q}} \subseteq \mathcal{U}$. Also $\mathfrak{Q}_{\mathfrak{q}}$ contains all real constants (including $-\infty$ ) and is closed under linear combinations with nonnegative rational coefficients. Our objective is to extend $\mathfrak{Q}_{\mathfrak{q}}$ in $\mathcal{U}$ by "closing" it with respect to a special closure operation defined on subsets of $\mathcal{U}$. We begin by defining two preliminary closure operations acting on an arbitrary subset $\mathcal{F}$ of $\mathcal{U}$. 
(1) Denote by $\mathcal{F}^{\downarrow}$ the set of all functions in $\mathcal{U}$ that are locally the pointwise limits of nonincreasing sequences of functions belonging to $\mathfrak{F}$.

(2) Denote by $\mathcal{F}^{s}$ the set of all functions in $\mathcal{U}$ that are locally the supremum of functions from $\mathfrak{F}$.

Note that the requirement that elements of $\mathcal{F}^{\downarrow}$ belong to $\mathcal{U}$ is automatically satisfied. It is also automatic in the case of $\mathfrak{F}^{s}$ if the function involved is locally the supremum of only a finite number of functions from $\mathcal{F}$. For arbitrary $\mathcal{F}$ the sets $\mathfrak{F}^{\prime}$ and $\mathcal{F}^{s}$ are always closed under restrictions. It is also easy to see that, if $\mathcal{F}$ is closed under addition, then the same is true of $\mathcal{F}^{\downarrow}$ and $\mathcal{F}^{s}$. If $\mathcal{F}$ is closed under addition of constants and multiplication by all nonnegative rationals, then $\mathcal{F} l$ and $\mathfrak{F}^{s}$ are closed under multiplication by all nonnegative reals.

The operations "f" and "s", as well as the operation of "local extension" used in defining $\mathfrak{U}$-holomorphic functions, are closure operations in the sense of the following general definition: Let $S$ be an arbitrary abstract set of points and let ${ }^{*}: X \rightarrow X^{*}$ be a mapping which associates with each subset $X$ of $S$ another subset $X^{*}$. Then " *" is called a closure operation in $S$ if it satisfies the following properties:

(i) $\emptyset^{*}=\varnothing(\varnothing$ the empty set $)$.

(ii) $X \subseteq X^{*}$ for every $X \subseteq S$.

(iii) $X_{1} \subseteq X_{2}$ implies $X_{1}^{*} \subseteq X_{2}^{*}$.

The closure operation is said to be proper if it satisfies the additional property

(iv) $\left(X^{*}\right)^{*}=X^{*}$, for every $X \subseteq S$.

A set $X$ is said to be ${ }^{*}$-closed if $X^{*}=X$. Note that both $\emptyset$ and $S$ are *-closed. Also, the intersection of an arbitrary collection of *-closed sets is ${ }^{*}$-closed. Therefore every subset of $S$ is contained in a smallest*-closed set, viz. the intersection of all *-closed sets that contain it. The smallest ${ }^{*}$-closed set that contains a given set $X$ is called the *-closure of $X$ and denoted by $X^{(*)}$. Note that " (*)," is a proper closure operation.

If "**1" and "**2" are two closure operations in $S$, then the composition "**1*2", where $X^{*} 1^{*} 2=\left(X^{*} 1\right)^{* 2}$, is also a closure operation in $S$. Moreover, it is easy to verify that $X^{*} 1^{*} 2=X$ if and only if both $X^{*} 1=X$ and $X^{*} 2=X$. Therefore $X^{*} 1^{*} 2=X$ if and only if $X^{*} 2^{*} 1=X$. It follows, in particular, that $\left(*^{*}{ }^{*}\right)=(* 2 * 1)$.

Returning to the two closure operations " $\downarrow$ ", and "s" in $\mathcal{U}$, introduced above, we consider the composite operations " $1 s$ " and "sl". Since it is the associated proper closure operations that are important, we may, by the above remark, restrict attention to "s $\downarrow$ ".

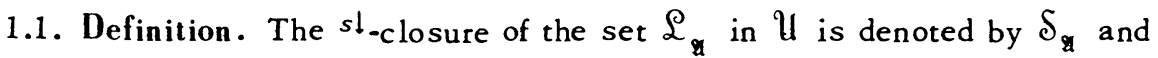
its elements are called $\mathfrak{U}$-subbarmonic functions. A function $f$ is called $\mathfrak{U}$-harmonic 
if both $f$ and $-f$ are $\mathfrak{U}$-subharmonic. The set of all $\mathfrak{U}$-harmonic functions is denoted by $\mathcal{H}_{\mathfrak{a}}$.

By the preceding remarks, the set $\mathcal{S}_{\mathscr{q}}$ is closed with respect to each of the

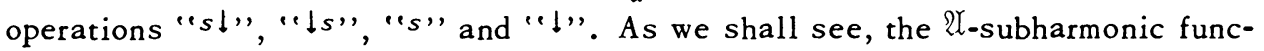
tions are a natural generalization of plurisubharmonic functions in $\mathbf{C}^{n}$.

The notation used in Definition 1.1 appropriately emphasizes the dependence of the sets $\mathfrak{L}_{\mathfrak{q}}, \mathcal{S}_{\mathfrak{q}}$ and $\mathcal{H}_{\mathfrak{q}}$ on the given system [ $\left.\Sigma, \mathfrak{X}\right]$. However, in the following discussion, we shall omit the subscript when no confusion can result. The set of all $\mathfrak{X}$-subharmonic functions defined on a set $\Omega \subseteq \Sigma$ is denoted by $\mathcal{S}(\Omega)$. The subset of $\mathcal{S}(\Omega)$ consisting of continuous functions is denoted by $C \mathcal{S}(\Omega)$. Similarly, we denote by $\mathcal{H}(\Omega)$ the subset of $\mathcal{S}(\Omega)$ consisting of $\mathcal{Y}$-harmonic functions. Note that $\mathcal{H}(\Omega) \subseteq C \mathcal{S}(\Omega)$ and elements of $\mathcal{H}(\Omega)$ do not assume infinite values.

The following proposition is very useful in the study of $\mathcal{S}$. It is formulated for the closure operation "s $s$ " but is valid for an arbitrary closure operation "*,". The proof, which involves a straightforward transfinite induction, will be omitted. (See $[5$, p. 191] or $[11$, p. 279].)

1.2. Proposition. There exists an ordinal $\mu$ such that to each $\nu \leq \mu$ there corresponds a set $\mathcal{S}_{\nu} \subseteq \mathcal{U}$ with the following properties:

(i) $\mathcal{S}_{0}=\mathfrak{L}, \mathcal{S}_{\mu}=\mathcal{S}$, and $\mathfrak{S}_{\alpha} \subsetneq \mathcal{S}_{\beta}$ for $0 \leq \alpha<\beta \leq \mu$.

(ii) If $\nu \leq \mu$, then $\mathcal{S}_{\nu}=\left(\bigcup_{a<\nu} \mathcal{S}_{\alpha}\right)^{s !}$.

With this decomposition of $\mathcal{S}$, we can use induction arguments to establish properties of the $\mathfrak{U}$-subharmonic functions. In particular, any property that involves only a finite number of functions and is satisfied in $\delta_{\nu}$ for some $\nu$ will be satisfied in $\mathcal{S}$ if it is preserved by "s" and "l".

Since the set $\mathcal{S}_{0}$ contains the constants and is closed under linear combinations with nonnegative rational coefficients, the set $\mathcal{S}_{1}$ is closed under linear combinations with nonnegative real coefficients. Moreover, the latter property is clearly preserved under "s" and " $\downarrow$ ", Therefore $\mathcal{S}$ is closed under linear combinations with nonnegative real coefficients. The set $\mathcal{H}$ consists of functions with finite real values and is closed under linear combinations with arbitrary real coefficients. Observe also that a function will belong to $\mathcal{S}$ if and only if it belongs locally to $\mathcal{S}$. More generally, we have the following theorem.

1.3. Theorem. $\mathfrak{S}$ is closed under local uniform limits. (I.e. $\operatorname{loc} \mathfrak{S}=\mathfrak{S}$.)

Proof. Let $g$ be a function which is locally approximable by elements of $\mathcal{S}$. Then, for each $\delta \in \mathscr{T}_{g}$, there exists a neighborhood $N$ of $\delta$ and a sequence $\left\{f_{n}\right\} \subset \mathcal{S}$, with $N \cap \mathscr{D}_{g}^{g} \subseteq \mathscr{D}_{f_{n}}$, such that $\sigma \in N \cap \mathscr{D}_{g}$ implies

$$
f_{n}(\sigma)<-n \quad \text { if } g(\sigma)=-\infty
$$


and

$$
g(\sigma)-1 / n<f_{n}(\sigma)<g(\sigma)+1 / n \quad \text { if } g(\sigma) \neq-\infty .
$$

We show first that $g \in \mathcal{U}$. For this it is enough to show that $g \mid\left(N \cap \mathscr{D}_{g}\right) \in \mathcal{U}$. Therefore, let $\sigma_{0} \in N \cap \mathfrak{I}_{g}$ and assume $g\left(\sigma_{0}\right)<t$. Choose $n$ such that $-n<t-$ $1 / n$ and $g\left(\sigma_{0}\right)<t-2 / n$. Then we have $f_{n}\left(\sigma_{0}\right)<t-1 / n$. Since $f_{n} \in \mathcal{U}$, there exists a neighborhood $N^{\prime}$ of $\sigma_{0}$ such that $N^{\prime} \subset N$ and $\sigma \in N^{\prime} \cap \mathscr{D}_{\boldsymbol{g}}$ implies $f_{n}(\sigma)<t-1 / n$. Since $g(\sigma) \leq f_{n}(\sigma)+1 / n$ for all $\sigma \in N \cap \mathfrak{D}_{g}$, it follows that $g(\sigma)<t$ for $\sigma \in N^{\prime} \cap \mathfrak{D}_{\boldsymbol{g}}$. Therefore, $g \in \mathcal{U}$.

Observe next that if we set

$$
g_{k}(\sigma)=\max [g(\sigma),-k], \quad \sigma \in \mathbb{T},
$$

then $g_{k} \in \mathcal{U}, g(\sigma) \leq g_{k+1}(\sigma) \leq g_{k}(\sigma)$, for all $k$, and

$$
g(\sigma)=\lim _{k \rightarrow \infty} g_{k}(\sigma), \quad \sigma \in \mathfrak{D}_{g} .
$$

Therefore, if we show that $g_{k} \in \mathcal{S}$ for each $k$, then it will follow that $g \in \mathcal{S}^{\downarrow}=\mathcal{S}$. Set

$$
f_{n k}(\sigma)=\max \left[f_{n}(\sigma),-k\right], \quad \sigma \in \mathbb{D}_{f_{n}}
$$

then $f_{n k} \in$ S. Also, $g(\sigma)=-\infty$ implies $g_{k}(\sigma)=-k$ and $f_{n k}(\sigma)=-k$ for $n \geq k$. Hence, if $n \geq k$, then $g_{k}(\sigma)-1 / n<f_{n k}(\sigma)<g_{k}(\sigma)+1 / n$ for all $\sigma \in N \cap \mathfrak{D}_{g}$. Since $f_{n k}-1 / n \in \mathcal{S}$ and

$$
g_{k}(\sigma)=\sup _{n}\left\{f_{n k}(\sigma)-1 / n\right\}, \quad \sigma \in N \cap T_{g},
$$

it follows that $g_{k} \in \mathcal{S}^{s}=\mathcal{S}$, so $g \in \mathcal{S}$, completing the proof of the theorem.

Consider next a real-valued function $\chi$ defined on an, possibly infinite, interval $(a, b)$. Assume that $\chi$ is nondecreasing and convex. Thus, if $a<r<s<b$, then $\chi(r) \leq \chi(s)$ and

$$
\chi((1-\theta) r+\theta s) \leq(1-\theta) \chi(r)+\theta \chi(s)
$$

for $0 \leq \theta \leq 1$. The function $\chi$ is automatically continuous on $(a, b)$ and, for convenience, we extend $\chi$ continuously to the half-open interval $[a, b)$ by setting

$$
\chi(a)=\lim _{t \rightarrow a^{+}} \chi^{(t)}
$$

Thus $\chi(a) \leq \chi(t)$ for all $t \in(a, b)$ and, if $a=-\infty$, then $\chi(a)=-\infty$ is possible. It follows from the convexity that the limit

$$
m_{t}=\lim _{s \rightarrow t^{+}} \frac{\chi^{(s)}-\chi^{(t)}}{s-t}
$$

exists for all $t \in(a, b)$ and that the graph of $\chi$ lies above the line through the 
point $(t, \chi(t))$ with slope equal to $m_{t}$, i.e. $\chi(t)+m_{t}(s-t) \leq \chi(s), s \in(a, b)$. Since $X$ is nondecreasing, $m_{t} \geq 0$ for all $t$.

1.4. Theorem. Let $f \in \mathcal{S}$ and assume that the range of values of $f$ is contained in the balf-open interval $[a, b)$ on which the nondecreasing, convex function $\chi$ is defined. Then $\chi \circ f \in \mathcal{S}$.

Proof. Observe first that since $\chi$ is continuous it follows that $\chi \circ f \in \mathcal{U}$. Also, since $\mathcal{S}$ contains the constants and $m_{t} \geq 0$, the function $\chi(t)+m_{t}(f-t)$ belongs to $\mathcal{S}$ for each $t \in(a, b)$. Therefore, if

$$
g_{t}(\sigma)=\max \left[\chi(a), \chi(t)+m_{t}(f(\sigma)-t)\right],
$$

for $\sigma \in \mathfrak{D}_{f}$, then also $g_{t} \in \mathcal{S}$. Moreover, for each $t \in(a, b), g_{t}(\sigma) \leq \chi(f(\sigma))$, $\sigma \in \mathscr{D}_{f}$. If $f(\sigma)=a$, then $g_{t}:=\chi(f(\sigma))$ for every $t$. If $f(\sigma) \in(a, b)$, then $g_{t}(\sigma)=$ $\chi(f(\sigma))$, for $t=f(\sigma)$. Therefore, $\sup _{t} g_{t}(\sigma)=\chi(f(\sigma)), \sigma \in \mathcal{D}_{f}$, so $\chi \circ f \in \mathcal{S}^{s}=\mathcal{S}$, completing the proof.

1.5. Corollary. (i) If $f \in \mathcal{S}$ and $f \geq 0$, then $f^{t} \in \mathcal{S}$ for arbitrary real $t \geq 1$.

(ii) If $f \in \mathcal{S}$, then $e^{f} \in \mathcal{S}$. In particular, if $g$ is logaritbmically $\mathscr{U}$-subbarmonic (i.e. $g \geq 0$ and $\log g \in \mathcal{S}$ ), then $g \in \mathcal{S}$.

(iii) For arbitrary $a \in \mathfrak{U}$, both $\log |a|$ and $|a|$ belong to $\mathcal{S}$.

We now extend (iii) of Corollary 1.5 to $\mathfrak{X}$-holomorphic functions.

1.6. Theorem. If $b$ is an arbitrary $\mathfrak{A}$-bolomorpbic function then both $\log |b|$ and $|b|$ belong to $\mathcal{S}$.

Proof. Our problem is to show that $\left|\mathscr{S}_{2}\right| \subseteq \mathcal{S}$ and $\log \left|\mathscr{F}_{2}\right| \subseteq \mathcal{S}$ where $\mathcal{F}_{2}$ is the family of all $\mathfrak{U}$-holomorphic functions in $\Sigma$. Denote by $\mathfrak{F}$ the family of all $b \in \mathcal{F}$ such that both $|b| \in \mathcal{S}$ and $\log |b| \in \mathcal{S}$. Then $\mathcal{F}$ is maximal in $\mathcal{F}$ with respect to the property that $|\mathfrak{F}| \subseteq \mathcal{S}$ and $\log |\mathcal{F}| \subseteq \mathcal{S}$. We must prove that $\mathcal{F}=\mathcal{S}$. Since $\mathfrak{U} \subseteq \mathcal{F}$, by Corollary 1.5 (iii), and $\mathscr{F}$ is the smallest locally closed family containing $\mathscr{A}$, it will be sufficient to prove that loc $\mathcal{F}=\mathcal{F}$ or, in view of the maximal character of $\mathcal{F}$, that $|\operatorname{loc} \mathcal{F}| \subseteq \mathcal{S}$ and $\log |\operatorname{loc} \mathcal{F}| \subseteq \mathcal{S}$.

Observe first that $|\operatorname{loc} \mathfrak{F}| \subseteq \operatorname{loc}|\mathfrak{F}| \subseteq \mathcal{S}$, where the last inclusion is given by Theorem 1.3. That $\log |\operatorname{loc} \mathcal{F}| \subseteq \mathcal{S}$ is more difficult. Let $b \in \operatorname{loc} \mathcal{F}$. Then, for arbitrary $\delta \in \mathcal{D}_{b}$, there exists a neighborhood $V$ of $\delta$ and $\left\{f_{n}\right\} \subset \mathcal{F}$ such that $\lim f_{n}(\sigma)=b(\sigma)$ uniformly on $V \cap \mathfrak{D}_{b}$. For an arbitrary positive integer $k$, define

$$
|b|_{k}(\sigma)=\max \left(e^{-k},|h(\sigma)|\right), \quad \sigma \in T_{h},
$$

and

$$
\left|f_{n}\right|_{k}(\sigma)=\max \left(e^{-i k},\left|f_{n}(\sigma)\right|\right), \quad \sigma \in V \cap T_{b} .
$$


Then $\lim \left|f_{n}\right|_{k}(\sigma)=|b|_{k}(\sigma)$ uniformly on $V \cap \mathscr{D}_{b}$. Moreover since the functions $\left\{\left|f_{n}\right|_{k}\right\}$ and $|b|_{k}$ are uniformly bounded away from zero for fixed $k$, we also have

$$
\lim _{n \rightarrow \infty} \log \left|f_{n}\right|_{k}(\sigma)=\log |b|_{k}(\sigma)
$$

uniformly on $V \cap \mathfrak{I}_{b}$. Observe next that

$$
\log \left|f_{n}\right|_{k}(\sigma)=\max \left(-k, \log \left|f_{n}(\sigma)\right|\right), \quad \sigma \in V \cap \mathfrak{I}_{b} .
$$

Since $\mathcal{S}$ contains the constants and $\log \left|f_{n}\right| \in \mathcal{S}$, it follows that $\log \left|f_{n}\right|_{k} \in \mathcal{S}^{s}=\mathcal{S}$. This implies that $\log |b|_{k} \in \operatorname{loc} \mathcal{S}$. Hence $\log |b|_{k} \in \mathcal{S}$, by Theorem 1.3. Finally observe that $\log |b| \leq \log |b|_{k+1} \leq \log |b|_{k}$, for all $k$, and

$$
\lim _{k \rightarrow \infty} \log |b|_{k}(\sigma)=\log |b(\sigma)|, \quad \sigma \in \mathscr{T}_{b} .
$$

Therefore, $\log |b| \in \mathcal{S}^{\mathfrak{l}}=\mathcal{S}$. Thus $\log |\operatorname{loc} \mathcal{F}| \subseteq \mathcal{S}$ and the proof is complete.

1.7. Corollary. Let $b$ be an arbitrary $\mathfrak{A}$-holomorpbic function.

(i) If $b$ is never zero, then $\log |b|$ is 0 -harmonic.

(ii) If $b=u+i v$, where $u$ and $v$ are real, then $u$ and $v$ are $\mathcal{U}-$ barmonic.

In the introduction it was pointed out that the obvious generalization to $[\Sigma, \mathfrak{U}]$ of the definition for plurisubharmonic functions in $\mathrm{C}^{n}$ was in some cases not restrictive enough. It is important to know, however, that our $\mathfrak{U}$-subharmonic functions do always satisfy the general definition even though the condition involved is not in all cases sufficient for a function to be $\mathscr{U}$-subharmonic. It will be convenient to consider holomorphic maps, not only of the unit disc, but also of an arbitrary open set of the plane into $\Sigma$. Thus if $U$ is an open set in $\mathbf{C}$ and $\eta$ maps $U$ into $\Sigma$, then $\eta$ is bolomorpbic if $a \circ \eta$ is holomorphic in the ordinary sense on $U$ for every $a \in \mathfrak{U}$. Note that such an $\eta$ is automatically continuous. Also, it is not difficult to prove that, if $b$ is any $\mathscr{U}$-holomorphic function defined on $\eta(U)$, then $b \circ \eta$ is an ordinary holomorphic function on $U$.

1.8. Theorem. Let $\eta$ be an arbitrary bolomorpbic mapping of an open set $U$ of $\mathrm{C}$ into $\Sigma$ and let $f$ be an arbitrary $\mathcal{U}$-subbarmonic function defined on $\eta(U)$. Then $f \circ \eta$ is subbarmonic in the ordinary sense on $U$.

Proof. Observe that if $f \in \mathscr{L}$, then $f=n^{-1} \log |a|$, where $a \in \mathfrak{U}$, so $f \circ \eta=$ $n^{-1} \log |a \circ \eta|$. By hypothesis, $a \circ \eta$ is holomorphic and hence $f \circ \eta$ is subharmonic on $U$. Therefore, the theorem is true for elements of $\mathscr{\&}$ and every choice of $\eta$ and $U$. Now denote by $\mathcal{F}$ the subset of $\mathcal{S}$ consisting of all those functions for which the theorem is true. In other words, if $f \in \mathcal{F}$ and $\eta$ is any holomorphic map of an open set $U$ into $\Sigma$ such that $\eta(U) \subseteq \mathscr{T}_{f}$, then $f \circ \eta$ is subharmonic on $U$. We thus have $\mathcal{L} \subseteq \mathcal{F} \subseteq \mathcal{S}$ and must prove that $\mathcal{F}=\mathcal{S}$. Since $\mathcal{S}$ is the smallest 
$s \downarrow$-closed subset of $\mathcal{U}$ that contains $\mathcal{L}$, it will be sufficient to prove that $\mathcal{F}^{s}=$ $\mathcal{F}=\mathfrak{F}^{\downarrow}$. Therefore let $g$ be an arbitrary element of either $\mathcal{F}^{s}$ or $\mathcal{F}^{\downarrow}$ and let $\eta$ be a holomorphic map of $U \subseteq \mathbf{C}$ into $\Sigma$ such that $\eta(U) \subseteq \mathfrak{D}_{\mathbf{g}}$. Since $g \in \mathcal{U}$ and $\eta$ is continuous, $g \circ \eta$ is u.s.c. on $U$. Also let $\zeta_{0}$ be an arbitrary point of $U$. Then $\eta\left(\zeta_{0}\right) \in \mathscr{D}_{g}$.

Assume first that $g \in \mathcal{F}^{s}$. Then there exists a neighborhood $N$ of $\eta\left(\zeta_{0}\right)$ such that

$$
g(\sigma)=\sup \left\{f(\sigma): f \in \mathcal{F}, N \cap \mathfrak{D}_{g} \subseteq \mathfrak{T}_{f}, f \leq g \text { on } N \cap \mathfrak{D}_{g}\right\},
$$

for each $\sigma \in N \cap \mathscr{D}_{g}$. Since $\eta$ is continuous, there exists a neighborhood $V$ of $\zeta_{0}$ in $U$ such that $\eta(V) \subseteq N \cap \mathscr{D}_{g}$. By hypothesis, if $f \in \mathcal{F}$ and $N \cap \mathscr{D}_{g} \subseteq \mathscr{D}_{f}$, then $f \circ \eta$ is subharmonic on $V$. Also, if $f \leq g$ on $N \cap \mathscr{D}_{g}$, then $f \circ \eta \leq g \circ \eta$ on $V$. Therefore, $g \circ \eta$ is the supremum on $V$ of subharmonic functions. Hence $g \circ \eta$ is subharmonic on $V[6$, Theorem 1.6.2]. Thus $g \circ \eta$ is locally subharmonic and is therefore subharmonic on $U$. This completes the proof that $\mathfrak{F}^{s}=\mathfrak{F}$.

Next assume that $g \in \mathcal{F} !$ Then there exists a neighborhood $N$ of $\eta\left(\zeta_{0}\right)$ and $\left\{f_{n}\right\} \subset \mathcal{F}$ such that $N \cap \mathscr{D}_{g} \subseteq \mathscr{D}_{f_{n}^{\prime}} g(\sigma) \leq f_{n+1}(\sigma) \leq f_{n}(\sigma)$, for all $n$ and $\sigma \in N \cap \mathscr{D}_{g}$, and $\lim f_{n}(\sigma)=g(\sigma)$ for $\sigma \in N \cap \mathfrak{D}_{g}$. Choose a neighborhood $V$ of $\zeta_{0}$ such that $\eta(V) \subseteq N \cap \mathscr{D}_{g}$. Then $f_{n} \circ \eta$ is subharmonic on $V$ for each $n$. Also, for $\zeta \in V$, $(g \circ \eta)(\zeta) \leq\left(f_{n+1} \circ \eta\right)(\zeta) \leq\left(f_{n} \circ \eta\right)(\zeta)$ and $\lim \left(f_{n} \circ \eta\right)(\zeta)=(g \circ \eta)(\zeta)$. Again it follows that $g \circ \eta$ is subharmonic on $V$ [6, Theorem 1.6.2] and, be ing locally subharmonic, is subharmonic on $U$. This proves that $\mathfrak{F}^{\downarrow}=\mathcal{F}$ and completes the proof of the theorem.

It follows from the above theorem that, in the case of $\left[C^{n}, \Re\right]$, a $\Re$-subharmonic function defined on an open set in $\mathrm{C}^{n}$ is automatically plurisubharmonic. The converse, that every plurisubharmonic function is $\$$-subharmonic, is also true but nontrivial. The proof depends on two well-known approximation theorems for plurisubharmonic functions. (See, for example, [4, Theorems 13.91 and 13.10].) First let $P S$ denote the set of all plurisubharmonic functions defined on open sets in $\mathbf{C}^{n}$ and let $C P S$ denote the subset of $P S$ consisting of continuous functions. Also denote by $L$ the set of all functions of the form $n^{-1} \log |b|$, where $n$ is a positive integer and $b$ is defined and holomorphic on some open set in $\mathbf{C}^{n}$. Now consider a function $f \in P S$ with an open set $G$ as its domain of definition. Then, by the first approximation theorem, if $H \subset \subset G$, there exists a sequence $\left\{f_{n}\right\}$ of continuous (in fact, $k$-times continuously differentiable) plurisubharmonic functions on $H$ such that $f_{n}(\zeta) \geq f_{n+1}(\zeta) \geq f(\zeta)$ and $f_{n}(\zeta) \rightarrow f(\zeta)$ for $\zeta \in H$. This implies immediately that $P S \subseteq(C P S)^{\downarrow}$. Now, by the second approximation theorem, if $U$ is a domain of holomorphy in $\mathrm{C}^{n}$ and $f$ is an element of CPS defined on $U$, then, for $V \subset \subset U$ and each $\zeta \in V, f(\zeta)=\sup \{g(\zeta): g \in L, g \leq f$ on $V\}$. Since 
each point of $\mathbf{C}^{n}$ admits arbitrarily small neighborhoods that are domains of holomorphy, it follows immediately that $C P S \subseteq L^{s}$. Combining these results, and using Theorem 1.6, we have $P S \subseteq(C P S)^{\downarrow} \subseteq L^{s \downarrow} \subseteq \delta_{\mathfrak{P}}$, which, with Theorem 1.8, completes the proof of the following theorem.

1.9. Theorem. In $\left[\mathrm{C}^{n}, \Re\right]$, a function defined on an open set is plurisubbar-

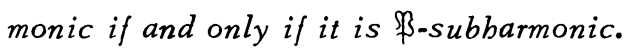

2. Maximum properties of $\mathfrak{U}$-subharmonic functions. Let $K$ be a compact subset of $\Sigma$ and $\mathcal{F}$ a collection of u.s.c. functions defined on $K$. A closed subset $B$ of $K$ is called an $\mathcal{F}_{\text {-set }}$ if

$$
\max _{\sigma \in B} f(\sigma)=\max _{\sigma \in K} f(\sigma)
$$

for every $f \in \mathcal{F}$. A simple Zorn's lemma argument shows that there always exists

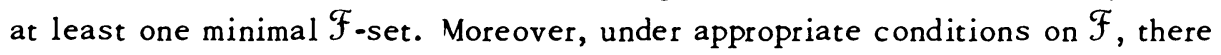
will exist a unique minimal $\mathcal{F}_{\text {oset, }}$ which we call the $\zeta_{i l o v}$ boundary of $K$ relative to $\mathcal{F}$ and denote by $\partial_{\mathcal{F}} K$. Sufficient conditions for uniqueness are that $\mathcal{F}$ be closed under addition and contain the constants along with enough functions to determine the topology on $K$. In particular, it is sufficient for $\mathfrak{F}$ to be closed under addition and contain $\log |\mathfrak{U}|$ (restricted to $K$ ). The proof of the existence of $\partial_{\mathcal{F}} K$ under these conditions is a straightforward adaptation of the proof for the existence of the Šilov boundary for an algebra of functions [3, Theorem 15.1]. The Silov boundary of $K$ relative to $\mathcal{S}(K)$ thus exists and we denote it simply by $\partial_{\delta} K$. Also, since $\mathcal{S}$ contains $\mathcal{L}$, it is easy to see that $\partial_{\delta} K$ contains the ordin-

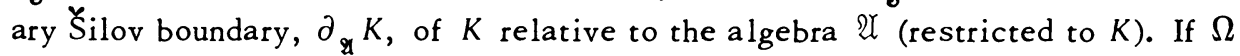
is a compact $\mathfrak{U}$-convex set, then, just as in the case of the $\mathfrak{U}_{\text {-holomorphic func- }}$ tions [11, Lemma 2.5], it turns out that $\partial_{\mathcal{S}} \Omega=\partial_{\Omega} \Omega$. This is a nontrivial fact which depends on local maximum properties of the $\mathfrak{X}$-subharmonic functions that we we shall now develop.

As might be expected, local maximum properties of $\mathfrak{A}$-subharmonic functions rest ultimately on the Rossi local maximum modulus principle for Banach algebras [13, Theorem 6.1]. We shall use the following special case of the Rossi principle: Let $\Omega$ be a compact $\mathfrak{U}$-convex subset of $\Sigma$ and let $U$ be a relatively open subset of $\Omega \backslash \partial_{\mathfrak{q}} \Omega$. Then, for every $a \in \mathfrak{U}$,

$$
\max _{\sigma \in \mathrm{bd}_{S_{\iota}} U}|a(\sigma)|=\max _{\sigma \in \bar{\Pi}}|a(\sigma)| \text {. }
$$

It is worth noting here that, in order for the Rossi principle to hold in this situation, $\Omega$ must be the space of maximal ideals (spectrum) of the algebra $\mathfrak{U}_{\Omega}$ obtained by closing $\mathfrak{U} \mid \Omega$ in $C(\Omega)$. This is equivalent to the condition that $[\Omega, \mathscr{U}]$ 
be natural, which in turn, since $[\Sigma, \mathfrak{O}]$ is assumed to be natural, is equivalent to the condition that $\Omega$ be $\mathscr{U}$-convex [11, Proposition 1.3].

For the discussion which follows, it will be convenient to introduce some definitions which generalize the above situation. First we define a subset of $\Sigma$ to be essentially open if it is a relatively open subset of some closed set in $\Sigma$ (e.g. the set $\Omega \backslash \partial_{\mathscr{Q}} \Omega$ above). Thus an essentially open set is simply the intersection of an open set and a closed set in $\Sigma$. If a set is essentially open, then it is obviously a relatively open subset of its closure. In general, we will denote an esentially open set by $\Delta_{0}$ and its closure by $\Delta$. We also set $\Gamma=\Delta \backslash_{0}$ and note that $\Gamma$ is equal to the boundary of $\Delta_{0}$ in $\Delta$.

Now let $\Delta_{0}$ be an essentially open set with compact closure $\Delta$ and let $\mathfrak{F}$ be a given subset of $\mathcal{U}$. Then $\mathcal{F}$ is said to satisfy the local maximum principle in $\Delta_{0}$ and $\Delta_{0}$ is said to be $\mathcal{F}$-local if for every set $U \subseteq \Delta_{0}$, relatively open in $\Delta_{0}$, and every $f \in \mathcal{U}$, with $\bar{U} \subseteq D_{\text {, }}$ and $f \mid U \in \mathcal{F}$, it is true that

$$
\max _{\sigma \in \mathrm{bd} \Delta U} f(\sigma)=\max _{\sigma \in \bar{U}} f(\sigma) .
$$

If $\Delta_{0}$ is $\mathfrak{F}$-local, then every relatively open subset of $\Delta_{0}$ is also $\mathcal{F}$-local.

Let $\mathcal{C}$ be a family of complex-valued functions and denote by $|\mathcal{C}|$ the family of absolute values of functions in $\mathcal{C}$. Then the set $\Delta_{0}$ is said to be $\mathcal{C}$-local provided it is $|\mathcal{C}|$-local. Thus if $\Omega$ is compact and $\mathscr{U}$-convex in $\Sigma$, then $\Omega \backslash \partial_{\Omega} \Omega$ is $\mathfrak{U}$-local. A fundamental property of $\mathfrak{U}$-subharmonic functions is that $\mathfrak{U}$-local implies $\mathcal{S}$-local. Before establishing this fact, we prove a localization lemma.

2.1. Lemma. Let $\Delta_{0}$ be essentially open with compact closure and let $U$ be a relatively open subset of $\Delta_{0^{*}}$. Also let $g$ be an element of $\mathcal{U}$ such that $\bar{U} \subseteq \mathfrak{D}_{g}$ and

$$
\max _{\sigma \in \mathrm{bd} \Delta U} g(\sigma)<0=\max _{\sigma \in \bar{U}} g(\sigma) .
$$

Then there exists a point $\delta \in U$ with the following property: For every neighborbood $N$ of $\delta$, there is a nonnegative integer $m$ and an element $u \in \mathfrak{U}$ such that, if $g_{N}=m g+\log |u|$, then

$$
\max _{\sigma \in \mathrm{bd}_{\Delta}(N \cap U)} g_{N}(\sigma)<0=g_{N}(\delta) .
$$

Proof. Consider the set $Z=\{\sigma \in \bar{U}: g(\sigma)=0\}$. By hypothesis, $Z \subset U$ and, since $g$ is u.s.c., $Z$ is closed and hence compact. Take $\delta$ to be any strong boundary point (see $[9,3.3 .15]$ ) of $Z$ relative to the algebra $\mathfrak{U}_{Z}$ obtained by closing $\mathfrak{X} \mid Z$ in $C(Z)$. Let $N$ be an arbitrary open neighborhood of $\delta$. If $Z \subset N$, then the function $g_{N}=g$ (i.e. take $m=1$ and $u=1$ ) already has the desired property. If $Z \notin N$, then, since $\delta$ is a strong boundary point, there exists $u \in \mathscr{U}$ 
such that $\max _{\sigma \in Z \backslash N}|u(\sigma)|<e^{-1}<|u(\delta)|=1$, so

$$
\max _{\sigma \in Z \backslash N} \log |u(\sigma)|<-1<\log |u(\delta)|=0 .
$$

Set $V=\{\sigma \in U: \log |u(\sigma)|<-1\}$. Then $V$ is relatively open in $\Delta_{0}$ and $Z \backslash N \subset V$. Next set $B=\left[\operatorname{bd}_{\Delta}(N \cap U)\right] \backslash V$. Then $B$ is compact and $Z \cap B=\varnothing$. If $B=\varnothing$, then $b_{\Delta}(N \cap U) \subset V$ and the function $g_{N}=\log |u|$ (i.e. take $m=0$ ) has the desired property. Therefore assume $B \neq \emptyset$ and set $s=\max _{\sigma \epsilon B} g(\sigma)$. Since $Z \cap B=\varnothing$, it follows that $s<0$. Therefore there exists a positive integer $m$ such that $m s+\log |u|_{\Delta}<0$, where $|u|_{\Delta}=\max \{|u(\sigma)|: \sigma \in \Delta\}$. Now let $g_{N}=$ $m g+\log |u|$. Then clearly $g_{N}(\delta)=0$. Furthermore, for $\sigma \in B=\operatorname{bd}_{\Delta}(N \cap U) \backslash V$, $g_{N}(\sigma) \leq m s+\log |u|_{\Delta}<0$ and, for $\sigma \in V \cap \operatorname{bd}_{\Delta}(N \cap U), g_{N}(\sigma)<m \cdot 0+(-1)=-1$. Therefore,

$$
\max _{\sigma \in \mathrm{bd}_{\Delta}(N \cap U)} g_{N}(\sigma)<0
$$

and the lemma follows.

2.2. Theorem. If an essentially open set with compact closure is $\mathfrak{X}$-local, then it is also $\mathcal{S}$-local.

Proof. Assume that the given essentially open set $\Delta_{0}$ is $\mathscr{H}$-local. Then, since the logarithm is increasing and $\mathcal{S}_{0}=\mathfrak{L}=\log |\mathfrak{U}|$, the set $\Delta_{0}$ is also $\mathcal{S}_{0^{-}}$ local. Next let $\mathcal{F}$ be any set of functions with $\mathcal{S}_{0} \subseteq \mathcal{F} \subseteq \mathcal{U}$, which is closed under addition, and denote by $\mathcal{G}$ either $\mathfrak{F}^{s}$ or $\mathfrak{F}^{\downarrow}$. Note that $\mathcal{G}$ is also closed under addition. Now, by Proposition 1.2, the desired result will follow by induction if we show that $\mathcal{F}$-local implies $\mathcal{G}$-local for the set $\Delta_{0}$. Therefore suppose, on the contrary, that $\Delta_{0}$ is $\mathcal{F}$-local but not $\mathcal{G}$-local. Then, using the fact that $\mathcal{G}$ contains all real constants (since $\mathcal{\delta}_{0} \subseteq \mathcal{G}$ ) and is closed under addition, we can obtain $U$, relatively open in $\Delta_{0}$, and a $g \in \mathcal{U}$ such that $\bar{U} \subseteq \mathscr{D}_{g}, g \mid U \in \mathcal{S}$, and

$$
\max _{\sigma \in \mathrm{bd}} U(\sigma)<0=\max _{\sigma \in \bar{U}} g(\sigma) \text {. }
$$

Thus Lemma 2.1 applies. Let $\delta$ be the point of $U$ given by the lemma. Since $g \mid U \in \mathcal{G}$ and $\mathcal{S}$ is either $\mathcal{F}^{s}$ or $\mathcal{F}^{\downarrow}$, there exists a neighborhood $N_{\delta}$ of $\delta$ such that, on the set $N_{\delta} \cap \mathscr{I}_{\mathcal{G}}$, the function $g$ is determined by $\mathcal{F}$ in accordance with the definition of $\mathcal{F}^{s}$ or $\mathfrak{F}^{\prime}$. Choose a smaller neighborhood $N$ of $\delta$ such that $\overline{N \cap \Delta_{0}} \subset N_{\delta}$ and let $g_{N}$ be the corresponding function given by Lemma 2.1. Thus $g_{N}=m g+\log |u|$, where $m$ is a nonnegative integer and $u \in \mathfrak{U}$, and we can choose $r$ so that

$$
\max _{\sigma \in \mathrm{bd}_{\Delta}(N \cap U)} g_{N}(\sigma)<r<0=g_{N}(\delta)
$$

Now assume that $\mathcal{G}=\mathcal{F}^{s}$. Then, for each $\sigma \in N_{\delta} \cap \mathscr{D}_{\boldsymbol{g}}$, 


$$
g(\sigma)=\sup \left\{f(\sigma): f \in \mathcal{F}, f \leq g \text { on } N_{\delta} \cap \mathfrak{D}_{g}\right\} .
$$

For arbitrary $f \in \mathcal{F}$ with $f \leq g$ on $N_{\delta} \cap \mathscr{X}_{g}$, set $f_{N}=m f+\log |u|$. Then $f_{N} \leq g_{N}$ on $N_{\delta} \cap \mathscr{D}_{\boldsymbol{g}}$ and, for each $\sigma \in N_{\delta} \cap \mathscr{D}_{\boldsymbol{g}}$,

$$
g_{N}(\sigma)=\sup \left\{f_{N}(\sigma): f \in \mathcal{F}, f \leq g \text { on } N_{\delta} \cap \mathfrak{D}_{g}\right\} .
$$

Hence we can choose $f \in \mathcal{F}$, with $f \leq g$ on $N_{\delta} \cap \mathscr{D}_{g}$, such that $r<f_{N}(\delta)$. Since $f_{N} \leq g_{N}$ on $N_{\delta} \cap \mathscr{D}_{g}$, and hence on $\operatorname{bd}_{\Delta}(N \cap U)$, it follows that

$$
\max _{\sigma \in \mathrm{bd}_{\Delta}(N \cap U)} f_{N}(\sigma)<f_{N}(\delta) \leq \max _{\sigma \in \overline{\mathrm{N}^{\prime} \mathrm{n} U}} f_{N}(\sigma) .
$$

This contradicts the assumption that $\Delta_{0}$ is $\mathcal{F}$-local and completes the proof for $\mathfrak{G}=\mathfrak{F}^{s}$.

Now assume $\mathcal{G}=\mathfrak{F} \downarrow$. Then there exists a sequence $\left\{f_{n}\right\} \subset \mathcal{F}$ such that, for all $n, \mathscr{D}_{g} \subseteq \mathscr{D}_{f_{n}}, g \leq f_{n+1} \leq f_{n}$, and $\left\{f_{n}\right\}$ converges pointwise to $g$ on $N_{\delta} \cap \mathscr{D}_{g}$. For each $n$, define $f_{n}^{\prime}=m f_{n}+\log |u|$. Then $\left\{f_{n}^{\prime}\right\} \subset \mathcal{F}, g_{N} \leq f_{n+1}^{\prime} \leq f_{n}^{\prime}$, and $\left\{f_{n}^{\prime}\right\}$ converges pointwise to $g_{N}$ on $N_{\delta} \cap \mathscr{D}_{g}$. Note that $\emptyset \neq \operatorname{bd}_{\Delta}(N \cap U) \subset N_{\delta} \cap \mathfrak{D}_{g}$. Hence, for each $\sigma \in \operatorname{bd}_{\Delta}(N \cap U)$, there exists $n_{\sigma}$ such that $n \geq n_{\sigma}$ implies $f_{n}^{\prime}(\sigma)<r$. Since $f_{n}^{\prime}$ is u.s.c., there exists a neighborhood $N_{\sigma}$ of $\sigma$ such that $f_{n_{\sigma}}^{\prime}\left(\sigma^{\prime}\right)<r$ for $\sigma^{\prime} \in N_{\sigma} \cap \mathfrak{I}_{g}$. The set bd ${ }_{\Delta}(N \cap U)$ is compact so is covered by a finite number of the neighborhoods $N_{\sigma}$, say $\left\{N_{\sigma_{i}}: i=1, \cdots, k\right\}$.

Let $n_{0}=\max \left\{n_{\sigma_{1}}, \cdots, n_{\sigma_{k}}\right\}$. Then

$$
f_{n_{0}}^{\prime} \leq f_{n_{\sigma_{i}}} \text { on } N_{\sigma_{i}} \cap \mathscr{D}_{g} \text { for each } i,
$$

so $f_{n_{0}}^{\prime}(\sigma)<r, \sigma \in \operatorname{bd}_{\Delta}(N \cap U)$. Moreover, $0=g_{N}(\delta) \leq f_{n_{0}}^{\prime}(\delta)$ and $\delta \in N \cap U$ so

$$
\max _{\sigma \in \mathrm{bd} \Delta(N \cap U)} f_{n_{0}}^{\prime}(\sigma)<\max _{\sigma \in \bar{N} \overline{n U}} f_{n_{0}}^{\prime}(\sigma) .
$$

This contradicts the assumption that $\Delta_{0}$ is $\mathcal{F}$-local and completes the proof of the theorem.

2.3. Corollary. If $\Omega$ is a compact $\mathfrak{A}$-convex set, then $\partial_{\Omega} \Omega=\partial_{\delta} \Omega$.

For the next theorem recall that a point $\delta$ in $\Sigma$ is called an independent point of $[\Sigma, \mathfrak{X}]$ if $\delta \in \hat{K^{\wedge}}$, for any compact set $K \subseteq \Sigma$, implies $\delta \in K$, where $K^{\wedge}$ denotes as usual the (necessarily compact) $\mathfrak{A}$-hull of $K$ in $\Sigma[11, \mathrm{p} .275]$. The point $\delta$ is said to be locally independent if there exists a neighborhood $N_{\delta}$ of $\delta$ such that $\delta$ is an independent point of $\left[N_{\delta}, \mathfrak{U}\right]$. By a general version of the local maximum modulus principle, a point of $\Sigma$ is independent if and only if it is locally independent [11, Theorem 1.8]. Note that $\left[\mathrm{C}^{n}, \Re\right]$ has no independent points.

2.4. Theorem. Let $f$ be $\mathfrak{U}$-subbarmonic on an open set $G \subseteq \Sigma$. If $\delta$ is any point of $G$ wbich is not an independent point of $[\Sigma, \mathfrak{X}]$, then 
Proof. Set

$$
f(\delta)=\limsup _{\sigma \rightarrow \delta} f(\sigma)
$$

$$
s=\lim _{\sigma \rightarrow \delta} \sup _{\delta} f(\sigma)=\inf _{N_{\delta}} \sup _{\sigma \in N_{\delta}^{\prime}} f(\sigma),
$$

where $N_{\delta}$ ranges over all neighborhoods of $\delta$ contained in $G$ and $N_{\delta}^{\prime}$ denotes the deleted neighborhood $N_{\delta} \backslash\{\delta\}$. Since $f$ is u.s.c., we have immediately that $s \leq$ $f(\delta)$. Since $\delta$ is not locally independent and points of $\Sigma$ admit arbitrarily small Ul-convex neighborhoods, there exists for each $N_{\delta}$ a compact set $K \subset N_{\delta}$, with $K^{\wedge} \subset N_{\delta}$, such that $\delta \in K^{\wedge}$ but $\delta \notin K$. We have, by Corollary $2.3, \partial_{\delta} K^{\wedge}=$ $\partial_{\mathrm{a}} K^{\wedge} \subseteq K$. Therefore,

$$
f(\delta) \leq \max _{\sigma \in K} f(\sigma) \leq \sup _{\sigma \in N_{\delta}^{\prime}} f(\sigma)
$$

Since $N_{\delta}$ can be arbitrarily small, it follows that $f(\delta) \leq s$, completing the proof of the theorem.

2.5. Corollary. Let $\Omega$ be a compact $\mathfrak{U}$-convex set in $\Sigma$ and let $f$ be $\mathfrak{U}$ subbarmonic on $\Omega$. Then, for every $\delta \in \Omega \backslash \partial_{\mathfrak{\imath}} \Omega$,

$$
f(\delta)=\lim _{\sigma \rightarrow \delta} \sup _{\delta} f(\sigma) \text {. }
$$

In the following theorem, which generalizes a well-known result for ordinary subharmonic functions, the set $\Delta_{0}$ is again essentially open with compact closure and is $\mathfrak{U}$-local. Since the strong local maximum modulus principle holds for holomorphic functions defined on open sets in $\mathrm{C}^{n}$, the set $\Delta_{0}$, for the special case $\left[\mathrm{C}^{n}, \mathfrak{\beta}\right]$, could be an arbitrary bounded open subset of $\mathbf{C}^{n}$.

2.6. Theorem. Let $U$ be a relatively open subset of $\Delta_{0}$ and let $h, g$ be functions such that $b$ is continuous on $\bar{U}$ and $\mathfrak{U}$-harmonic on $U$ while $g$ is u.s.c. on $\bar{U}$ and $\mathscr{U}$-subbarmonic on $U$. Then $g \leq b$ on bd $U$ implies $g \leq b$ on $\bar{U}$.

Proof. By the definition of $\mathfrak{A}$-harmonicity, both $b$ and $-b$ are continuous on $\bar{U}$ and $\mathscr{U}$-subharmonic on $U$. Therefore, the function $g-b$ is u.s.c. on $\bar{U}$ and U-subharmonic on $U$. By hypothesis, $g-b \leq 0$ on bd bd $_{\Delta}$. Since $\Delta_{0}$ is $\mathfrak{A}$-local it is also $\mathcal{S}$-local, by Theorem 2.2 , so it follows that $g-b \leq 0$ on $\bar{U}$. Hence, $g \leq b$ on $\bar{U}$ and the theorem is proved.

2.7. Corollary. If $g$ and $b$ are both 2 -harmonic on $U$, then $g=b$ on $\operatorname{bd}_{\Delta} U$ implies $g=b$ on $\bar{U}$.

3. Convexity with respect to $\mathscr{U}$-subharmonic functions. Let $G$ be an arbitrary open subset of $\Sigma$ and consider the set $C \mathscr{S}(G)$ of all continuous $\mathfrak{U}$-subharmonic functions defined on $G$. (The value $-\infty$ is permitted for elements of $C \delta(G)$.) Also 
let $\mathcal{F}$ be an arbitrary subset of $C \mathscr{S}(G)$. For a compact set $K \subset G$, we define the $\mathcal{F}$-bull of $K$, in the usual way, to be the set

$$
K_{G}^{\mathfrak{F}}=\left\{\sigma \in G: f(\sigma) \leq \max _{\delta \in K} f(\delta), f \in \mathcal{F}\right\} .
$$

Since elements of $\mathcal{F}$ are continuous, the set $K_{G}^{\mathcal{F}}$ is relatively closed in $G$. If $K \subseteq H \subseteq G$, then the intersection of $K_{G}^{\mathcal{F}}$ with the set $H$ will be denoted by $K_{G, H^{*}}^{\mathcal{F}}$. The set $K_{G, H}^{\mathfrak{F}}$ is called the $\mathcal{F}$-bull of $K$ in $H$.

3.1. Definition. If $K_{G}^{\mathcal{F}}$ is compact for every compact set $K \subset G$, then $G$ is

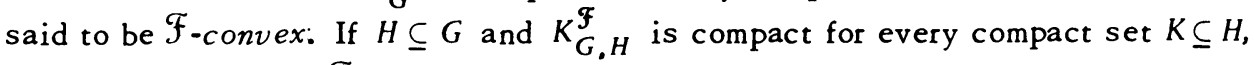
then $H$ is said to be $\mathcal{F}$-convex in $G$.

For the special case $\mathcal{F}=C \mathscr{S}(G)$, the $C \mathscr{S}(G)$-hulls are called "थO-sh. bulls" and denoted by $K_{G}^{\Pi}$ and $K_{G, H}^{\Pi}$. Also, in this case, we use the term "थH-sh. con. vexity" in place of " $C \mathcal{S}(G)$-convexity".

If $\mathcal{F}=|\mathcal{C}|$, where $\mathcal{C}$ is a family of complex-valued functions defined on $G$, then $\mathcal{F}$-convexity reduces to the usual $\mathcal{C}$-convexity. In the special case, $\mathcal{C}=$ 2I $G$, we denote the $\mathcal{C}$-hull of the compact set $K \subset G$ by $\hat{K_{G}}$. Note that $\hat{K_{G}}=$ $\hat{K \cap} \cap G$, where $K^{\wedge}$ is the $\mathfrak{U}$-hull of $K$ in $\Sigma$. If $\hat{K_{G}}$ is compact, then $\hat{K^{\wedge}}=\hat{K_{G}} U$ $(K \backslash G)$ is a decomposition of the $\mathscr{U}$-convex set $K^{\wedge}$ into disjoint closed sets, each of which, by a general result, must be $\mathfrak{U}$-convex [11, Proposition 1.4]. Since $K \subseteq \hat{K_{G}}$, it follows that $\hat{K^{\wedge}}=\hat{K_{G}}$. Therefore, $\mathfrak{X} \mid G$-convexity of $G$ is already equivalent to $\mathfrak{U}$-convexity of $G$ in $\Sigma$. This implies that $\mathfrak{X} \mid G$-convexity of an open set $H$ in $G$ is also equivalent to $\mathscr{H}$-convexity of $H$ in $\Sigma$.

In the special case, $\mathcal{C}=\hat{C}_{G}$, where $\hat{C}_{G}$ is the family of all $\mathfrak{U}$-holomorphic functions on $G$, we use the terminology "थh-holomorpbic convexity", in place of " $C_{G}$-convexity", and denote the hulls $K_{G}^{\left|\theta_{G}\right|}$ and $K_{G, H}^{\left|\theta_{G \mid}\right|}$ respectively by $K_{G}$ and $K_{G, H}$. In the usual finite-dimensional cases, Ut-holomorphic convexity reduces to ordinary holomorphic convexity and $\mathfrak{U}$-sh. convexity reduces to $p$-convexity, as defined by Gunning and Rossi [4, p. 276]. Since, by Theorem 1.6, $|\mathfrak{X}| G|\subseteq| \mathcal{O}_{G} \mid \subseteq C \mathfrak{S}(G)$, it follows that $K_{G}^{n} \subseteq K_{G}^{\bigcap} \subseteq K_{G} \subseteq K^{\hat{K}}$, so the se sets have compact closures. Also we observe that $\mathfrak{U}$-convexity of $G$ implies $\mathfrak{A}$-holomorphic convexity which in turn implies $\mathscr{U}$-sh. convexity. We shall explore in more detail the relationship between $\mathfrak{U}$-holomorphic convexity and $\mathfrak{U}$-sh. convexity in the next section. In this section we develop some of the basic properties of U-sh. convexity.

Let $G$ be an open subset of $\Sigma$. For $u \in C \mathfrak{S}(G)$ and real $t$, set $G(u<t)=$ $\{\sigma \in G: u(\sigma)<t\}$. Then $G\left(u<t_{1}\right) \subseteq G\left(u<t_{2}\right)$, for $t_{1}<t_{2}$, and $G=\bigcup G(u<t)$. Let $K$ be a compact subset of $G(u<t)$ and, for convenience, set $K_{t}=K_{G}^{\Pi} \cap$ $G(u<t)=K_{G}^{\Gamma}, G(u<t)$. Since $K_{G}^{\Pi}$ is relatively closed in $G$ and $K_{t} \subseteq K_{G}^{\Pi}$, it follows that $\bar{K}_{t} \cap G \subseteq K_{G}^{\top}$. Moreover, since $u$ is continuous and $K \subset G(u<t)$, we have 


$$
\sup _{\sigma \in \mathbb{R}_{t} \cap G} u(\sigma)=\sup _{\sigma \in K_{t}} u(\sigma)=\max _{\sigma \in K} u(\sigma)<t,
$$

so $\bar{K}_{t} \cap G \subset G(u<t)$. Therefore $\bar{K}_{t} \cap G=K_{t}$. Observe that the set $K_{t}$ will be compact if and only if it is closed and will be closed if and only if $\bar{K}_{t} \subset G$. It follows that $G(u<t)$ will be $\mathfrak{U}$-sh. convex in $G$ if and only if $\bar{K}_{t} \subset G$ for every compact set $K$ in $G(u<t)$.

3.2. Theorem. (i) If either $G$ is $\mathscr{X}$-sh. convex or $\overline{G(u<t)} \subset G$, then $G(u<t)$ is $\mathfrak{A}$-sh. convex within $G$.

(ii) If $\overline{G(u<t)} \subset G$ for all real $t$, then $G$ is $\mathcal{U}-s h$. convex.

Proof. For the proof of (i), let $K$ be an arbitrary compact subset of $G(u<t)$ and observe that

$$
\bar{K}_{t}=\overline{K_{G}^{\Pi} \cap G(u<t)} \subseteq \overline{K_{G}^{\bar{T}}} \cap \overline{G(u<t)}
$$

If $G$ is $\mathscr{X}$-sh. convex, then $K_{G}^{\Pi}$ is compact, so $\overline{K_{G}^{\Pi}}=K_{G} \subset G$ and hence $\bar{K}_{t} \subset G$. Also, if $\overline{G(u<t)} \subset G$, then $\bar{K}_{t} \subset G$. Therefore, in either case, $G(u<t)$ is $\mathscr{U}$-sh. convex within $G$, by the preceding remarks.

For the proof of (ii), let $K$ be an arbitrary compact subset of $G$ and choose $t>\max _{\sigma \in K} u(\sigma)$. Then

$$
K_{G}^{\Gamma} \subset G(u<t) \subset \overline{G(u<t)} \subset G .
$$

Since $K_{G}^{\Pi}$ is always relatively closed in $G$, it follows that $K_{G}^{\Pi}$ is in fact closed and therefore compact. Thus $G$ is $\mathscr{U}$-sh. convex.

The results in the remainder of this section require compactness conditions on the open sets involved. The first result is a converse to part (ii) of the above theorem. (Compare [6, Theorem 2.6.7].)

3.3. Theorem. Let $G$ be an open, $\sigma \cdot c o m p a c t$ subset of $\Sigma$. If $G$ is 2 -sh. convex, then there exists $g \in C S(G)$ such that, for every real $t, G(g<t) \subset \subset G$.

Proof. Since $G$ is $\sigma$-compact, there exists a sequence $\left\{G_{n}\right\}$ of open sets such that $G_{n} \subset \subset G_{n+1}$, for all $n$, and $G=\bigcup G_{n}$. Since $G$ is also $\mathscr{U}$-sh. convex, we can assume further that $\bar{G}_{n}^{n} \subset G_{n+1}$. Set $F_{n}=\bar{G}_{n+1} \backslash G_{n}$. Then $\left\{F_{n}\right\}$ is a sequence of compact sets and

$$
G \backslash G_{n}=\bigcup_{k=n}^{\infty} F_{k} .
$$

Since $\bar{G}_{n}^{n} \subset G_{n+1}$, it follows that $\bar{G}_{n}^{n} \cap F_{n+1}=\emptyset$. Hence, for each $\delta \in F_{n+1}$, there exists $u_{\delta} \in C \delta(G)$ such that $\max _{\sigma \in \bar{G}_{n}} u_{\delta}(\sigma)<0<u_{\delta}(\delta)$. Since $u_{\delta}$ is continuous, there exists a neighborhood $V_{\delta}$ of $\delta$, with $V_{\delta} \subset G$, such that $\inf _{\sigma \in V_{\delta}} u(\sigma)>0$. Since $F_{n+1}$ is compact, it is covered by a finite number of the neighborhoods $V_{\delta}$, say $V_{\delta_{1}}, \cdots, V_{\delta_{m}}$. Define 


$$
v_{n}(\sigma)=\max \left\{u_{\delta_{1}}(\sigma), \cdots, u_{\delta_{m}}(\sigma)\right\}, \quad \sigma \in G .
$$

Then $v_{n} \in C \mathscr{S}(G)$ and

$$
\max _{\sigma \in \bar{G}_{n}} v_{n}(\sigma)<0<\min _{\sigma \in F_{n+1}} v_{n}(\sigma) .
$$

After modifying $v_{n}$ by a positive multiplicative constant, we can assume that $\max _{\sigma \in \bar{G}_{n}} v_{n}(\sigma)<-n \log 2$ and $\log (n+1)<\min _{\sigma \in F_{n+1}} v_{n}(\sigma)$. Now, replacing $v_{n}$ by $w_{n}=e^{v_{n}}$, we have $w_{n} \geq 0, w_{n} \in C S(G)$, and

$$
\max _{\sigma \in \bar{G}_{n}} w_{n}(\sigma)<1 / 2^{n}, \quad n+1<\min _{\sigma \in F} w_{n+1}(\sigma) .
$$

Finally, we define

$$
g(\sigma)=\sum_{k=1}^{\infty} w_{k}(\sigma), \quad \sigma \in G .
$$

If $k>n$, then $\bar{G}_{n} \subset G_{k}$. Therefore, for $\sigma \in G_{n}$,

$$
\sum_{k=n+1}^{\infty} w_{k}(\sigma)<\sum_{k=n+1}^{\infty} \frac{1}{2^{k}} .
$$

It follows that the series for $g$ converges uniformly on $G_{n}$. In particular, it converges locally uniformly in $G$, so $g \in C \mathscr{S}(G)$.

For arbitrary real $t$, choose $n \geq t$. Then, if $\delta \in G \backslash G_{n}$, there exists $m \geq n$ such that $\delta \in F_{m}$. Since the functions $w_{k}$ are positive, we have $g(\delta)=\sum_{k=1}^{\infty} w_{k}(\delta) \geq$ $w_{m-1}(\delta)>m \geq t$. Therefore, $G(g<t) \subseteq G_{n}$ and, since $G_{n} \subset \subset G$, it follows that $G(g<t) \subset \subset G$.

3.4. Corollary. If $G$ is an open $\sigma$-compact, $\mathfrak{A}-s h$. convex set in $\Sigma$, then there exists a sequence $\left\{G_{n}\right\}$ of open sets with $G_{n} \subset \subset G_{n+1} \subset \subset G=\bigcup_{k=1}^{\infty} G_{k}$ such that each $G_{n}$ is Û.sh. convex within $G$.

We shall obtain below, as a consequence of the next theorem, a converse to the result in Corollary 3.4. (See Theorem 3.7.)

3.5. Theorem. Let $G$ and $H$ be open sets in $\Sigma$, with $H \subseteq G$, where $G$ is locally compact and $H$ is $\mathscr{U}$-sh. convex within G. Then, for every compact set $K$ in $H, K K_{G}^{\Pi}$ is compact and contained in $H$.

Proof. Assume that $K$ is a compact set in $G$ such that $K_{G}^{\Pi}=F \cup F^{\prime}$, where $F$ is compact and $F \cap \bar{F}^{\prime}=\emptyset$. We shall prove that $F_{G}^{n}=F$.

Since $F$ is compact and $G$ is locally compact, there exists an open set $U$, with compact closure and hence a compact boundary, bd $U$ (possibly empty), such that $F \subset U \subset \subset G, \bar{F}^{\prime} \cap \bar{U}=\varnothing$. Note that bd $U \subset G \backslash K_{G}^{\Pi}$. If bd $U=\varnothing$, then we have immediately that $F_{G}^{\cap}=F$ (since the function $g$ such that $g(\sigma)=0$, for $\sigma \in U$, and $g(\sigma)=1$, for $\sigma \in G \backslash U$, belongs to $C \delta(G)$ ). Therefore, assume that bd $U \neq \varnothing$. 
For each $\delta \in$ bd $U$, there exists $f_{\delta} \in C S(G)$ with

$$
\max _{\sigma \in K_{G}^{n}} f_{\delta}(\sigma)=\max _{\sigma \in K} f_{\delta}(\sigma)<0<f_{\delta}(\delta) .
$$

Set $N_{\delta}=\left\{\sigma \in G: 0<f_{\delta}(\sigma)\right\}$. Then $N_{\delta}$ is an open neighborhood of $\delta$. Since bd $U$ is compact, it is covered by a finite collection of these neighborhoods. Denote this finite covering by $N_{1}, \cdots, N_{n}$ with associated functions $f_{1}, \cdots, f_{n}$. Let

$$
t_{0}=\max \left\{f_{i}(\sigma): \sigma \in K ; i=1, \cdots, n\right\} .
$$

Then $t_{0}<0$. Choose $t$ such that $t_{0}<t<0$, and set $V=\left\{\sigma \in G \backslash \bar{U}: f_{i}(\sigma)<t\right.$; $i=1, \cdots, n\}$. Then $V$ is open and contains $F^{\prime}$. Observe that for each $i, f_{i}(\sigma)>0$ if $\sigma \in($ bd $U) \cap N_{i}$ while $f_{i}(\sigma) \leq t<0$ if $\sigma \in \bar{V}$. Therefore, (bd $\left.U\right) \cap \bar{V}=\varnothing$ and hence $\bar{U} \cap \bar{V}=\varnothing$, so $G=(G \backslash \bar{U}) \cup(G \backslash \bar{V})$. Now define

$$
\begin{array}{ll}
g_{1}(\sigma)=\max \left\{f_{i}(\sigma): i=1, \cdots, n\right\}, & \sigma \in G, \\
g_{2}(\sigma)=\max \left\{t, g_{1}(\sigma)\right\}, & \sigma \in G .
\end{array}
$$

If $\sigma \in(G \backslash \bar{U}) \cap(G \backslash \bar{V})$, then $f_{i}(\sigma) \geq t$, for some $i$, so $g_{1}(\sigma)=g_{2}(\sigma)$. Therefore, if we let

$$
g(\sigma)= \begin{cases}g_{1}(\sigma), & \sigma \in G \backslash \bar{V}, \\ g_{2}(\sigma), & \sigma \in G \backslash \bar{U},\end{cases}
$$

then $g$ is well defined in $G$. Furthermore, since the constant $t$ and each of the functions $f_{i}$ belong to $C \mathscr{S}(G)$, it follows that $g \in C S(G)$. Observe next that $F \subset$ $U \subset G \backslash \bar{V}$ while $F^{\prime} \subset V \subset G \backslash \bar{U}$. Hence

$$
\max _{\sigma \in F} g(\sigma)=\max _{\sigma \in F} g_{1}(\sigma) \leq t_{0}<t,
$$

while, for each $\sigma \in F^{\prime}, g(\sigma)=g_{2}(\sigma) \geq t$. Therefore $F_{G}^{\Pi} \subset G \backslash F^{\prime}$. Furthermore, $F_{G}^{\mathbf{n}} \subseteq K_{G}^{\boldsymbol{\Pi}}$, so we conclude that $F \subseteq F_{G}^{\mathbf{\Pi}} \subseteq K_{G}^{\boldsymbol{\Pi}} \backslash F^{\prime}=F$. Therefore, $F_{G}^{\boldsymbol{\Pi}}=F$.

Now let $K$ be a compact subset of $H$. Then the set $K_{G, H}^{\Pi}=K_{G}^{\Pi} \cap H$ is compact so $K_{G}^{\Pi}=\left(K_{G}^{\Pi} \cap H\right) \cup\left(K_{G}^{\boldsymbol{\eta}} \backslash H\right)$ is a decomposition of $K_{G}^{\dot{\Pi}}$ of the type considered above. Therefore, since $K \subseteq K_{G}^{\Pi} \cap H$, it follows that $K_{G}^{\Pi}=K_{G}^{\Pi} \cap H$, completing the proof.

3.6. Corollary. If $G$ and $H$ are as in the theorem and if $H=H_{1} \cup H_{2}$, where $\bar{H}_{1} \cap H_{2}=H_{1} \cap \bar{H}_{2}=\varnothing$, then $H_{1}$ and $H_{2}$ are also $\mathfrak{A}$-sh. convex within $G$.

The following theorem is the promised converse to Corollary 3.4. An arbitrary collection of sets will be called increasing if the union of any finite subcollection of its elements is contained in an element of the collection. 
3.7. Theorem. Let $G$ be an open locally compact subset of $\Sigma$ which is the union of an increasing collection of open sets each of which is U-sh. convex within $G$. Then $G$ is $\mathfrak{A}-s h$. convex.

Proof. Let $K$ be a compact subset of $G$. Then there exists an element $H$ of the increasing collection of open sets which contains $K$. Since $H$ is 21 -sh. convex within $G$, Theorem 3.5 applies and we conclude that $K_{G}^{n}$ is compact. In other words, $G$ is $\mathfrak{U}$-sh. convex, as claimed.

We close this section with an approximation theorem.

3.8. Theorem. Let $G$ be a locally compact open set in $\Sigma$ and let $\Omega$ be a subset of $G$ which is compact and $\mathfrak{U}_{-s h}$. convex within $G$ (i.e. $\Omega_{G}^{n}=\Omega$ ). Then there exists an open set $H$, with $\Omega \subset H \subseteq G$, sucb that $H$ is $\mathcal{U}$-sh. convex within $G$.

Proof. Choose an open set $U$ such that $\Omega \subset U \subset \subset G$. Applying to the set $\Omega$ the same construction used with $F$ in the proof of Theorem 3.5, we obtain a function $g_{1} \in C \mathscr{S}(G)$ such that

$$
\max _{\sigma \in \boldsymbol{\Omega}} g_{1}(\sigma)<0<\min _{\sigma \in \mathrm{bd} U} g_{1}(\sigma)
$$

Now choose a real number $r$ such that $0<r<\min _{\sigma \in \mathrm{bd} U} g_{1}(\sigma)$, then $\Omega \subset U\left(g_{1}<0\right) \subset \subset$ $U\left(g_{1}<r\right) \subset U$, where $U\left(g_{1}<t\right)=\left\{\sigma \in U: g_{1}(\sigma)<t\right\}$. Observe that

$$
G=U\left(g_{1}<r\right) \cup\left(G \backslash \overline{U\left(g_{1}<0\right)}\right)
$$

and, if $\sigma \in U\left(g_{1}<r\right) \cap\left(G \backslash \overline{U\left(g_{1}<0\right)}\right)$, then $g_{1}(\sigma) \geq 0$. Next define

$$
g_{2}(\sigma)=\max \left\{0, g_{1}(\sigma)\right\}, \quad \sigma \in G .
$$

Then $g_{2} \in C \delta(G)$ and $g_{1}(\sigma)=g_{2}(\sigma)$ for $\sigma \in U\left(g_{1}<r\right) \cap\left(G \backslash \overline{U\left(g_{1}<0\right)}\right)$. Therefore, if

$$
g(\sigma)= \begin{cases}g_{1}(\sigma), & \sigma \in U\left(g_{1}<r\right), \\ g_{2}(\sigma), & \sigma \in G \backslash \overline{U\left(g_{1}<0\right)},\end{cases}
$$

then $g$ is well defined in $G$ and belongs to $C \mathscr{S}(G)$. Furthermore, $U\left(g_{1}<0\right)=$ $G(g<0)$. Since $\overline{G(g<0)} \subset G$, it follows by Theorem $3.2(\mathrm{i})$ that $G(g<0)$ is U-sh. convex within $G$. Therefore the theorem follows with $H=G(g<0)$.

4. U-subharmonic vs. Utholomorphic convexity. We have already observed that $\mathfrak{A}$-holomorphic convexity always implies $\mathfrak{U}$-sh. convexity. It is a fundamental convexity problem to obtain a converse to this statement. More precisely, if $G$ is an open set in $\Sigma$ and $H$ is a subset of $G$, then the problem is to obtain appropriate conditions on $G$, or on $H$, under which 2 -sh. convexity of $H$ within $G$ will imply $\mathscr{U}$-holomorphic convexity of $H$ within $G$. The most interesting, and also 
most difficult, case occurs when $H=G$. This is the problem of determining when $\mathfrak{U}$-sh. convexity implies $\mathscr{U}$-holomorphic convexity. In the case of $\left[\mathrm{C}^{n}, \mathfrak{B}\right]$, every $\Re$-sh. convex (i.e. $p$-convex) domain is holomorphically convex. This is also true more generally for Riemann domains [4, Theorem 4, p. 283]. Since convexity properties of this kind are already difficult to prove in these special cases, it is not suprising that we have been unable to generalize the more delicate results. Nevertheless, it is possible to obtain in the general case certain convexity theorems that are still nontrivial in finite dimensions. The proof of our first result, though short, involves most of the theory of natural systems. (Compare [ 8 , Proposition 3, p. 56] and [4, Theorem 16, p. 279].)

4.1. Theorem. Let $G$ be an open, 2T-bolomorpbically convex subset of $\Sigma$ and let $K$ be a compact subset of $G$. Then $K_{G}^{\boldsymbol{n}}=K_{G}^{n}$.

Proof. Observe first that, since $G$ is $\mathcal{X}$-holomorphically convex, the system $\left[G, \mathcal{C}_{G}\right]$ is natural [11, Theorem 3.5]. Therefore $K_{G}^{n}$ is a compact $\mathcal{C}_{G}$-convex subset of $G$. Note also that, since $\mathfrak{U} \mid G \subseteq \mathcal{C}_{G}$ and $\left|\mathcal{C}_{G}\right| \subseteq \mathcal{S}$, it follows that the class of all $C_{G}$-subharmonic functions in $G$ coincides with the class of all 2 subharmonic functions defined on subsets of $G$. Therefore, by Corollary 2.3, with $[\Sigma, \mathfrak{Q}]$ replaced by $\left[G, \mathcal{C}_{G}\right]$, we have

$$
\partial_{\delta} K_{G}=\partial_{O G} K_{G} \subseteq K .
$$

This implies $K_{G} \subseteq K_{G}^{\Pi}$ and, since $K_{G}^{\Pi} \subseteq K_{G}^{\bigcap}$ is always true, the theorem follows.

Remark. Note that the inclusion, $\partial_{S} K_{G} \subseteq K$, obtained in the above proof, implies a stronger conclusion than the one stated in the theorem; viz., the bull of $K$ in $G$ with respect to $\mathcal{S}(G)$ (rather than just $C \mathcal{S}(G)$ ) is equal to $K_{G}$. Using this fact, we obtain a generalization of a result due to Bremermann [2, Theorem 4].

4.2. Theorem. Let $G$ be an open, $\mathcal{U}$-holomorpbically convex subset of $\Sigma$ and let $S, T$ be subsets of $G$ with $S \cup T \subset \subset G$. If

$$
\max _{\sigma \in \bar{T}}|b(\sigma)|=\max _{\sigma \in \overline{S \cup T}}|b(\sigma)|
$$

for all $b \in \mathcal{C}_{G}$, then also

for all $f \in \mathcal{S}(G)$.

$$
\max _{\sigma \in \bar{T}} f(\sigma)=\max _{\sigma \in \overline{S U T}} f(\sigma)
$$

Proof. The hypothesis of the theorem is equivalent to the condition that the set $\overline{S \cup T}$ be contained in the $C_{G}$-hull of $\bar{T}$ in $G$. Therefore, by the above remark, $\overline{S \cup T}$ is contained in the $S(G)$-hull of $\bar{T}$ in $G$, which implies the desired result. 
4.3. Theorem. Let $G$ be an open $\mathscr{U}$-bolomorpbically convex subset of $\Sigma$ and let $H \subseteq G$. Then, in order for $H$ to be $\mathscr{U}$-bolomorpbically convex within $G$, it is necessary and sufficient that $H$ be $\mathfrak{U}$-sh. convex within $G$.

Proof. We have only to prove the sufficiency. Hence assume that $H$ is $\mathcal{U}$-sh. convex within $G$ and let $K$ be a compact subset of $H$. By Theorem 4.1, $K_{G}^{\Pi}=K_{G}^{\bigcap}$ so $K_{G, H}^{\bigcap}=K_{G}^{\bigcap} \cap H=K_{G}^{n} \cap H=K_{G, H}^{n}$. Since $H$ is $\mathscr{U}$-sh. convex, the set $K_{G, H}^{\Pi}$, and hence $K_{G, H}$, is compact. In other words, $H$ is $\mathscr{N}$-holomorphically convex within $G$, as desired.

The above theorem, along with Theorem 3.2, gives the following corollary.

4.4. Corollary. Let $u \in C S(G)$. Then, for arbitrary real $t$, the set $G(u<t)=$ $\{\sigma \in G: u(\sigma)<t\}$ is $\mathfrak{R}$-bolomorphically convex within $G$.

The same arguments used in the proofs of Theorems 4.1 and 4.3 may be used to establish a result for $\mathfrak{U}$-convexity.

4.5. Theorem. Let $H$ be an open set in $\Sigma$. Then a necessary and sufficient condition for $H$ to be $\mathfrak{U}$-convex is that it be $\mathfrak{U}$-sh. convex within $\Sigma$.

Proof. The necessity is obvious. Therefore assume that $H$ is $\mathfrak{A}$-sh. convex within $\Sigma$ and let $K$ be a compact subset of $H$. Then, exactly as in the proof of Theorem 4.1, we conclude that $K_{\Sigma}^{\mathbf{n}}=K^{\hat{\Lambda}}$. Since $H$ is $\mathfrak{A}$-sh. convex within $\Sigma$, the set $K_{\Sigma}^{\Gamma} \cap H$ is compact. Thus, $K^{\wedge}=\left(K^{\wedge} \cap H\right) \cup\left(K^{\wedge} \backslash H\right)$ is a decomposition of $K^{\wedge}$ into disjoint compact sets. Therefore, each of the sets $K^{\wedge} \cap H$ and $K^{\wedge} \backslash H$ is also -convex [11, Proposition 1.4]. Since $K \subseteq K^{\wedge} \cap H$, it follows that $K^{\wedge}=$ $K^{\wedge} \cap H \subset H$. Therefore $H$ is $\mathscr{U}_{-c o n v e x}$ and the theorem is proved.

It would be desirable to weaken, if possible, the condition on $G$ in Theorem 4.2. In this direction, we show that, even without any restrictions on $G$, one can still prove some local results involving certain concepts of "local convexity".

Any set $H$ is said to be $\mathfrak{U}$-convex at a point $\delta \in \Sigma$ if there exists an open neighborhood $V$ of $\delta$ such that $V \cap H$ is $\mathfrak{U}$-convex. If $H$ is $\mathfrak{U}$-convex at every point of $\Sigma$, then we say simply that $H$ is locally $\mathscr{U}$-convex. We also define in an analogous way the notions of $\mathfrak{U}$-bolomorpbic and $\mathfrak{U}$-sb. convexity at a point as well as local $\mathfrak{U}$-bolomorpbic and local $\mathfrak{U}$-sh. convexity. By contrast, we shall refer to the previous notions of convexity for $H$ as global convexity.

A consequence of the naturality of $[\Sigma, \mathfrak{U}]$ is that every point of $\Sigma$ has arbitrarily small open $\mathfrak{A}$-convex neighborhoods. It follows that the condition for an open set to be locally convex, in any of the above senses, is actually a condition on its boundary.

4.6. Proposition. If an open set $H$ is globally convex, in any of the above senses, then it is locally convex in the same sense. 
Proof. Let $\delta \in \Sigma$ and take $V$ to be an open $\mathfrak{A}$-convex neighborhood of $\delta$. If $K$ is a compact subset of $V \cap H$ and $K_{H}^{\sim}$ denotes the appropriate convex hull of $K$ in $H$, then we have $K_{V \cap H}^{\sim} \subseteq K_{H}^{\sim} \subseteq K \hat{\cap} H \subset V \cap H$. Therefore, if $K_{H}^{\sim}$ is compact, then $K_{V \cap H}^{\sim}$ is a compact subset of $V \cap H$, completing the proof.

In terms of the notion of one set being convex within another, we can formulate the following more restrictive definition of convexity at a point: An open set $H$ is said to be relatively convex at $\delta$ if there exists an open neighborhood $V$ of $\delta$ such that $V \cap H$ is convex within $V$. By the remarks following Definition 3.1, we already know that relative $\mathfrak{U}$-convexity at $\delta$ actually reduces to $\mathfrak{U}$-convexity at $\delta$, so there is nothing new. The following theorem is a much stronger result along the same lines.

4.7. Theorem. Relative $\mathfrak{A}$-sh. convexity (and hence also $\mathfrak{U}$-bolomorphic convexity) of an open set $H$ at a point $\delta$ is equivalent to $\mathfrak{U}$-convexity at $\delta$.

Proof. We must prove that relative $\mathfrak{U}$-sh. convexity implies $\mathfrak{A}$-convexity at $\delta$. Therefore let $V$ be a neighborhood of $\delta$ such that $V \cap H$ is $\mathscr{U}$-sh. convex within $V$. Let $U$ be an open $\mathscr{A}$-convex neighborhood of $\delta$ contained in $V$. If $K$ is a compact subset of $U \cap H$, then $K_{U \cap H, U}^{n} \subseteq K^{\wedge} \cap K_{V \cap H, V}^{n} \subset U \cap H$. By hypothesis, $K_{V \cap H, V}^{n}$ is compact so $K^{\wedge} \cap K_{V \cap H, V}^{n}$ is also compact. Hence $K_{U n H, U}^{n}$ is compact and we conclude that $U \cap H$ is $\mathscr{U}$-sh. convex within $U$. Therefore we may as well assume that $V$ is an open $\mathcal{U}$-convex neighborhood of $\delta$. Then the system $[V, \mathfrak{A}]$ (i.e. $[V, \mathfrak{A} \mid V]$ ) is natural [11, Proposition 1.3]. Observe also that $\mathcal{S}_{\mathfrak{q} \mid V}$ consists of those elements of $\mathcal{S}_{\mathfrak{q}}$ defined on subsets of $V$. Therefore U-sh. convexity of $V \cap H$ within $V$ is equivalent to $2 \mid V$-sh. convexity within $V$. Finally, an application of Theorem 4.5, with $[V, \mathfrak{O}]$ in place of $[\Sigma, \mathfrak{U}]$, enables us to conclude that $V \cap H$ is $\mathfrak{X} \mid V$-convex and hence $\mathfrak{A}$-convex, completing the proof of the theorem.

4.8. Corollary. Let $G$ and $H$ be open sets in $\Sigma$, where $H$ is $\mathfrak{U}$-sh. convex within $G$. Then $H$ is $\mathfrak{A}$-convex at each point of $G$. If $\bar{H} \subset G$, then $H$ is locally IY-convex.

Consider any open set $G$ and a function $u \in C \mathcal{S}(G)$. For real $t$, set $G(u<t)=\{\sigma \in G: u(\sigma)<t\}$ as in Theorem 3.2. Let $\delta \in G(u<t)$ and choose an open $\mathfrak{A}$-convex neighborhood $V$ of $\delta$ with $V \subset G$. Then $V$ is also $\mathfrak{U}$-sh. convex

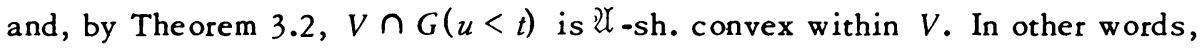
$G(u<t)$ is relatively $\mathfrak{A}$-sh. convex at $\delta$. Thus we have the following corollary.

4.9. Corollary. Let $u \in C \mathscr{S}(G)$, where $G$ is an arbitrary open set in $\Sigma$. Then, for each real $t$, the set $G(u<t)$ is U-convex at each point of $G$. If also $\overline{G(u<t)} \subset G$, then $G(u<t)$ is locally $\mathfrak{U}$-convex. 
A domain $G$ in $\mathbf{C}^{n}$ will be holomorphically convex ( $p$-convex) if and only if it is locally holomorphically convex (locally $p$-convex) [6, Theorem 2.6.10]. This is a nontrivial result and the problem of obtaining an adequate extension to the general case remains open. In fact, we are even unable to settle the weaker question as to when local $\mathfrak{U}$ convexity implies IT-holomorphic convexity.

We close with a result which involves a convexity property more reminiscent of the usual notion of convexity for a subset of a linear space.

Let $\Theta$ be an arbitrary subset of $\Sigma$. We call a point $\delta \in \Theta$ an inner point of $\Theta$ if it is not a locally independent point of the system $[\Theta, \mathfrak{U}]$. (See the remarks preceding Theorem 2.4.) This amounts to saying that if $V$ is any neighborhood of $\delta$, then there exists a compact set $K \subset V \cap \Theta$ such that $\delta \in K^{\wedge} \backslash K$. We shall also call a point $\delta \in \Theta$ an extension point of $\Theta$ if any function which is $\mathcal{U}$-holomorphic on a deleted neighborhood of $\delta$ within $\Theta$ has an 2 -holomorphic extension to the full neighborhood in $\Theta$. These notions, though formulated for an arbitrary set, are primarily of interest for a variety. For example, if $\Theta$ is an ordinary variety of $\operatorname{dim} \geq 2$ (say, in $\mathbf{C}^{n}$ ) then each of its points is an inner extension point. Finally we observe that, if $\Theta$ is an $\mathcal{U}$-analytic subvariety of an open set $U$ in $\Sigma$, [11, Definition 2.10], then it is $\mathscr{U}$-convex at each of its points. To see this, let $\delta \in \Theta$ and choose an open $\mathscr{U}$-convex neighborhood $V$ of $\delta$ with $V \subset U$. Then $V \cap \Theta$ is an $\mathfrak{A}$-analytic subvariety of the $\mathscr{U}$-convex set $V$ so is $\mathfrak{A}$-convex $[11$, Theorem 2.12].

4.10. Theorem. Let $G$ be an open set in $\Sigma$ and $\delta$ a point of $\Sigma$ at which $G$ is $\mathfrak{U}$-holomorphically convex. Also let $\Theta$ be a set which is $\mathfrak{U}$-convex at $\delta$ and for which $\delta$ is an inner extension point. Then $\Theta \backslash\{\delta\} \subset G$ implies $\Theta \subset G$ (i.e. $\delta \in G)$.

Proof. As in the proof of Theorem 4.7, we can choose an open 20 -convex neighborhood $V$ of $\delta$ such that $V \cap G$ is $\mathscr{U}$-holomorphically convex and $V \cap \Theta$ is $\mathscr{U}$-convex. Since $\delta$ is an inner point of $\Theta$, there exists a compact set $K C$ $V \cap \Theta$ such that $\delta \in K^{\wedge} \backslash K$. Note that $K^{\wedge} \subset V \cap \Theta$. Assume now that $\Theta \backslash\{\delta\}$ $C G$. Then $K \subset V \cap G$, so $K \hat{V \cap G}$ is a compact subset of $V \cap G$ that contains $K$. If it happens that $\delta \in K_{V} \hat{n G}$, then there is nothing to prove. Therefore assume that $\delta \notin K_{V \cap G}$. Then $K^{\wedge} \backslash K_{V \cap G}$ is a relatively open subset of $K^{\wedge}$ which contains $\delta$. Since $K^{\wedge}$ cannot contain an isolated point outside of $K$ [11, Proposition 1.4], it follows that the set $K^{\wedge} \backslash K_{V \cap G}^{\wedge}$ must contain points of $K^{\wedge}$ different from $\delta$. Let $\delta^{\prime}$ be one such point. Then

$$
\delta^{\prime} \in V \cap(\Theta \backslash\{\delta\}) \subset V \cap G .
$$

Hence there exists $b \in \mathcal{O}_{V \cap G}$ such that 


$$
\left|b\left(\delta^{\prime}\right)\right|>|b|_{K \hat{V} \cap G}=|b|_{K} .
$$

Note that the restriction of $b$ to $(V \cap \Theta) \backslash\{\delta\}$ is $\mathscr{A}$-holomorphic. Now since $\delta$ is an extension point of $\Theta, b(\delta)$ may be defined so that $b$ is $\mathscr{N}$-holomorphic on $V \cap \Theta$. In particular, $b$ is $\mathcal{U}$-holomorphic on $K^{\wedge}$. But then the inequality $\left|b^{\prime}\left(\delta^{\prime}\right)\right|>$ $|b|_{K}$ contradicts the local maximum principle for $\mathscr{U}$-holomorphic functions. Therefore it must be true that $\delta \in K_{V \cap G}$ so $\delta \in G$ and the proof is complete.

If in the above theorem $G$ is assumed to be $\mathscr{U}$-convex at $\delta$, then the assumption that $\Theta$ be 2 -convex at $\delta$ and that $\delta$ be an extension point for $\Theta$ can be dropped. To see this, proceed as before to obtain an open $\mathscr{U}$-convex neighborhood $V$ of $\delta$ such that $V \cap G$ is $\mathscr{H}$-convex, and a compact set $K \subset V \cap \Theta$ such that $\delta \in K^{\wedge} \backslash K$. If $\Theta \backslash\{\delta\} \subset G$, then $K \subset V \cap G$ so $K^{\wedge} \subset V \cap G$ and hence $\delta \equiv G$.

\section{REFERENCES}

1. S. Bochner and W. T. Martin, Several complex variables, Princeton Math. Series, vol. 10, Princeton Univ. Press, Princeton, N. J., 1948. MR 10, 366.

2. H.-J. Bremermann, On the conjecture of the equaivalence of the plurisubharmonic functions and the Hartogs functions, Math. Ann. 131 (1956), 76 -86. MR 17, 1070.

3. B. A. Fuks, Special chapters in the theory of analytic functions of several complex variables, Fizmatgiz, Moscow, 1963; Englısh transl., Transl. Math. Monographs, vol. 14, Amer. Math. Soc., Providence, R.I., 1965. MR 30 \#4979; MR 32 \#915.

4. R. C. Gunning and H. Rossi, Analytic functions of several complex variables, Prentice-Hall Series in Modern Analysis, Prentice-Hall, Englewood Cliffs, N. J., 1965. MR 31 \#4927.

5. F. Hausdorff, Mengenlehre, de Gruyter, Berlin, 1937; English transl., Set theory, Chelsea, New York, 1957. MR 19, 111.

6. L. Hörmander, An introduction to complex analysis in several variables, Van Nostr and, Princeton, N. J., 1966. MR 34 \#2933.

7. P. Lelong, Les fonctions plurisousharmoniques, Ann. Sci. École Norm. Sup. (3) 62 (1945), 301-338. MR 8, 271.

8. - Fonctions plurisousharmoniques et formes différentielles positives, Gordon and Breach, New York, 1968. MR 39 \#4436.

9. C. E. Rickart, General theory of Banach algebras, University Series in Higher Math., Van Nostrand, Princeton, N. J., 1960. MR 22 \#5903.

10. - Analytic phenomena in general function algebras, Pacific J. Math. 18 (1966), 361-377. MR 33 \#6438.

11. , Holomorphic convexity for general function algebras, Canad. J. Math. 20 (1968), 272-290. MR 37 \#3362.

12. - Analytic functions of an infinite number of complex variables, Duke Math. J. 36 (1969), 581-597. MR 40 \#7819.

13. H. Rossi, The local maximum modulus principle, Ann. of Math. (2) 72 (1960), 1-11. MR 22 \#317.

14. G. Stolzenberg, A hull with no analytic structure, J. Math. Mech. 12 (1963), 103111. MR $26 \# 627$. 